\title{
Dynamical Casimir Effect for a Swinging Cavity
}

\author{
Cem Yuce*, Zalihe Ozcakmakli \\ Department of Physics, Anadolu University, Eskisehir, Turkey. \\ cyuce@anadolu.edu.t.**
}

\begin{abstract}
The resonant scalar particle generation for a swinging cavity resonator in the Casimir vacuum is examined. It is shown that the number of particles grows exponentially when the cavity rotates at some specific external frequency.

PACS numbers: 03.65.Ge, 03.65.-w, 02.60.Lj
\end{abstract}

\section{INTRODUCTION}

In 1948, Casimir predicted an attractive force between two closely placed perfectly conducting plates due to the vacuum fluctuations [1]. The recent measurements confirm the basic concepts of quantum field theory in the presence of static external constraints [2]. The best way to learn more about the quantum vacuum is to distort the vacuum. One way to distort the vacuum is to vibrate one of the wall with one of the resonant field frequencies. Under this resonance condition, the vacuum field and the moving mirrors become strongly coupled to each other. The time dependent boundary conditions due to the moving mirrors chance the vacuum field in the cavity. As a result, it was theoretically predicted that photons are generated in the empty cavity because of the instability of the vacuum state of the electromagnetic field in the presence of time-dependent boundary conditions [3, 4, 5, 6, 7, 8, 9, 10, 11]. A number of virtual photons from the vacuum are converted into real photons. This phenomenon is known as dynamical Casimir effect or motion-induced radiation. There are a few proposed experimental for the detection of photons [12, 13, 14]. A one dimensional cavity with two perfectly parallel reflecting walls, one of which is motionless and the other oscillating with a mechanical frequency equal to a multiple of the fundamental optical resonance frequency of the static cavity, has been used as a simple model to study the dynamical Casimir effect [4, 5, $, \underline{6}, \underline{7}$ ].

$$
L_{1}(t)=0, \quad L_{2}(t)=L(1+\epsilon \sin \Omega t),
$$

where the constant $\Omega$ is the external frequency, $\epsilon$ is a small parameter, the constant $L$ is the initial length of the cavity and $L_{1}(t)$ and $L_{2}(t)$ are the positions of the right and the left walls at time $t$, respectively. The cavity is motionless initially and that at some instant one mirror starts to oscillate resonantly with a tiny amplitude. The case of cavities with two moving mirrors was studied by few authors 15, 16, 17, 18, 19, 20, 21, 22, 23]. Compared to the situation with a single oscillating mirror, it was found that radiation is resonantly enhanced when the cavity oscillates symmetrically with respect to the cavity center. It was also shown that photons are generated when the cavity oscillates as a whole, that is, the length of the cavity is constant [23].

So far the attention has been mainly focused on the oscillation of the mirrors of the cavity. Among the previous works on the quantum vacuum, there is a lack of information about the resonance photon generation for a rotating cavity in vacuum. In this paper, we will examine the resonance scalar particle generation when the cavity rotates around itself. To our knowledge, particle generation due to the rotation of the cavity was not considered before in the literature.

\section{SCALAR FIELD}

Consider a cubic cavity formed by perfect conductors whose sides have a length $L$. The scalar field vanishes on the surfaces. So, the Dirichlet boundary conditions read

$$
\Phi(x=\{0, L\}, y, z, t)=0, \quad \Phi(x, y=\{0, L\}, z, t)=0, \quad \Phi(x, y, z=\{0, L\}, t)=0,
$$

where the field operator in the Heisenberg representation obeys the wave equation, $c=1$

$$
\nabla^{2} \Phi=\frac{\partial^{2} \Phi}{\partial t^{2}}
$$

The field operator inside the cavity can be expanded as

$$
\Phi(\mathbf{x}, t)=\sum_{n}\left(b_{\mathbf{n}} \Psi_{\mathbf{n}}(\mathbf{x}, t)+b_{\mathbf{n}}^{\dagger} \Psi_{\mathbf{n}}^{\star}(\mathbf{x}, t)\right),
$$


where $b_{\mathbf{n}}^{\dagger}$ and $b_{\mathbf{n}}$ are the creation and the annihilation operators, respectively and $\Psi_{\mathbf{n}}(\mathbf{x}, t)$ is the corresponding mode function. For $t<0$, the mode function satisfying the boundary conditions is expanded as

$$
\Psi_{\mathbf{n}}=N_{\mathbf{n}} e^{-i \omega_{\mathbf{n}} t} \sin \left(\frac{n_{x} \pi x}{L}\right) \sin \left(\frac{n_{y} \pi y}{L}\right) \sin \left(\frac{n_{z} \pi z}{L}\right)
$$

where $\omega_{\mathbf{n}}=\pi / L \sqrt{n_{x}^{2}+n_{y}^{2}+n_{z}^{2}}$ and the constant $N_{\mathbf{n}}=(2 / L)^{\frac{3}{2}}\left(1 / \sqrt{2 \omega_{\mathbf{n}}}\right)$.

At time $t=0$, the cavity starts to rotate. In the literature, the most common and useful way to describe the rotation of a rigid body is to use the Euler angles. For the simplicity, assume that the $z$-axis lies along the axis of rotation. The problem looks simple, since the wave equation is the same as whether the boundaries are rotating or not. However, the boundary conditions become time-dependent. Although the solution of the wave equation is easy to be found and well known in static case (5), finding the exact solution of the same problem endowed with the time-dependent boundary conditions is very difficult.

In the following, we denote the fixed system in unprimed coordinates and the rotated one in primed coordinates. The orthogonal transformation matrix about the $z$-axis through the angle $\theta$ transforms the fixed unprimed axes to the primed axes leaving the distance between two points unchanged.

$$
\left(\begin{array}{l}
x^{\prime} \\
y^{\prime} \\
z^{\prime}
\end{array}\right)=\left(\begin{array}{ccc}
\cos \theta(t) & \sin \theta(t) & 0 \\
-\sin \theta(t) & \cos \theta(t) & 0 \\
0 & 0 & 1
\end{array}\right)\left(\begin{array}{l}
x \\
y \\
z
\end{array}\right)
$$

where $\dot{\theta}$ is the angular velocity and dot denotes time derivation with respect to $t$.

The boundary conditions in the rotating system is given by

$$
\Phi\left(x^{\prime}=\{0, L\}, y^{\prime}, z^{\prime}, t\right)=0, \quad \Phi\left(x^{\prime}, y^{\prime}=\{0, L\}, z^{\prime}, t\right)=0, \quad \Phi\left(x^{\prime}, y^{\prime}, z^{\prime}=\{0, L\}, t\right)=0 .
$$

One can also derive the time dependent boundary conditions by using (6).

We look for the rotations of the cavity that could enhance the number of generated photons by means of resonance effects for some specific external frequencies. To enhance the number of photons, let us now assume the resonance condition,

$$
\theta(t)=\epsilon \sin (\Omega t)
$$

where the constant $\Omega$ is the external frequency and the constant $\epsilon$ is a small parameter ( $L \dot{\theta}$ is very small compared to the speed of the light, where $L$ is the length).

There are two ways to deal with this problem. One can either solve the wave equation with the time dependent boundary conditions or transform the time dependent boundary conditions to the fixed boundary conditions. In the latter case, the form of the wave equation is changed. We will follow the second case. Let us now find the new form of the wave equation. The derivative operators transform according to $\nabla^{2} \rightarrow \nabla^{\prime 2}, \frac{\partial}{\partial t} \rightarrow \frac{\partial}{\partial t}-i \dot{\theta} J_{z}$ where $i J_{z}=x^{\prime} \frac{\partial}{\partial y^{\prime}}-y^{\prime} \frac{\partial}{\partial x^{\prime}}$. Substituting these into the wave equation (3) and neglecting the terms with $\dot{\theta}^{2}$ gives the transformed wave equation in the primed coordinate system.

$$
\nabla^{\prime 2} \Phi=\frac{\partial^{2} \Phi}{\partial t^{2}}-i J_{z}\left(2 \dot{\theta} \frac{\partial}{\partial t}+\ddot{\theta}\right) \Phi
$$

The last term in the right hand side is due to the rotation of the cavity. Now, we do not have to deal with the time dependent boundary conditions. However, we are left with a new equation.

We will try to solve this equation. To find the explicit form of the field operator, we will follow the approach given in [24, 25, 26]. For an arbitrary moment of time $t>0$, the mode function (4) satisfying the boundary conditions (7) is expanded as

$$
\Psi_{\mathbf{k}}=\sum_{\mathbf{n}} c_{\mathbf{n}}^{\mathbf{k}}(t)\left(\frac{2}{L}\right)^{\frac{3}{2}} \sin \left(\frac{n_{x} \pi x^{\prime}}{L}\right) \sin \left(\frac{n_{y} \pi y^{\prime}}{L}\right) \sin \left(\frac{n_{z} \pi z^{\prime}}{L}\right)
$$

where $c_{\mathbf{n}}^{\mathbf{k}}(t)=c_{n_{x} n_{y} n_{z}}^{k_{x} k_{y} k_{z}}(t)$ is a time-dependent function to be determined later. The initial conditions are given by $c_{\mathbf{n}}^{\mathbf{k}}(0)=1 / \sqrt{2 \omega_{\mathbf{k}}} \delta_{\mathbf{n}, \mathbf{k}}$ and $\dot{c}_{\mathbf{n}}^{\mathbf{k}}(0)=-i \sqrt{\omega_{\mathbf{k}} / 2} \delta_{\mathbf{n}, \mathbf{k}}$.

Let us substitute (10) into the equation (9) in order to find $c_{\mathbf{n}}^{\mathbf{k}}(t)$. If we multiply the resulting equation with 
$\sin \left(m_{x} \pi x^{\prime} / L\right) \sin \left(m_{y} \pi y^{\prime} / L\right) \sin \left(m_{z} \pi z^{\prime} / L\right)$ and use the orthogonality relations, we get an infinite set of coupled differential equations for $c_{\mathbf{n}}^{\mathbf{k}}$ after some algebra.

$$
\ddot{c}_{\mathbf{m}}^{\mathbf{k}}+\omega_{\mathbf{m}}^{2} c_{\mathbf{m}}^{\mathbf{k}}=\epsilon \delta_{n_{z} m_{z}}\left(8 \sum_{n_{x}} \sum_{n_{y}} g_{n_{y} m_{y}}^{n_{x} m_{x}} \Theta_{\mathbf{n}}^{\mathbf{k}}+\sum_{n_{x}} g_{n_{x} m_{x}} \Theta_{\mathbf{n}}^{\mathbf{k}} \delta_{n_{y} m_{y}}-\sum_{n_{y}} g_{n_{y} m_{y}} \Theta_{\mathbf{n}}^{\mathbf{k}} \delta_{n_{x} m_{x}}\right)
$$

where $g_{n m}=\frac{\left(1-(-1)^{m+n}\right) m n}{m^{2}-n^{2}}, \quad g_{n_{y} m_{y}}^{n_{x} m_{x}}=\frac{1}{\pi^{2}}\left(\frac{1}{m_{x}^{2}-n_{x}^{2}}-\frac{1}{m_{y}^{2}-n_{y}^{2}}\right) g_{n_{x} m_{x}} g_{n_{y} m_{y}}$ and they are equal to zero when $n_{x}=m_{x}$ or $n_{y}=m_{y} . \Theta_{\mathbf{n}}^{\mathbf{k}}$ is defined as

$$
\epsilon \Theta_{\mathbf{n}}^{\mathbf{k}}=\left(2 \dot{\theta} \dot{c}_{\mathbf{n}}^{\mathbf{k}}+\ddot{\theta} c_{\mathbf{n}}^{\mathbf{k}}\right)
$$

We will try to solve the second order differential equation (11). Conventional weak-coupling perturbation theory suffers from problems that arise from resonant terms in the perturbation series. The effects of the resonance could be insignificant on short time scales but become important on long time scales. Perturbation methods generally break down after small time whenever there is a resonance that lead to what are called secular terms. In the equation (11), this happens for those particular values of external frequency $\Omega$ such that there is a resonant coupling with the eigenfrequencies of the static cavity. A number of approaches have been developed to solve such equations. For example, averaging over fast oscillations [27, 29], multiple scale analysis [30, 31], the rotating wave approximation [32], numerical techniques [33]. We prefer to use the multiple scale analysis method (MSA), a powerful and sophisticated perturbative method valid for longer times.

In MSA, the trick is to introduce a new variable $\tau=\epsilon t$. This variable is called the slow time because it does not become significant in the small time. The functional dependence of $c_{\mathbf{m}}^{\mathbf{k}}$ on $t$ and $\epsilon$ is not disjoint because it depends on the combination of $\epsilon t$ as well as on the individual $t$ and $\epsilon$. The time variables $t$ and $\tau$ are treated independently in MSA. Thus, in place of $c_{\mathbf{m}}^{\mathbf{k}}(t)$, we write $c_{\mathbf{m}}^{\mathbf{k}}(t, \epsilon t)$. Let us expand $c_{\mathbf{m}}^{\mathbf{k}}$ in the form of a power series in $\epsilon$

$$
c_{\mathbf{m}}^{\mathbf{k}}(t)=c_{\mathbf{m}}^{\mathbf{k}(0)}(t, \tau)+\epsilon c_{\mathbf{m}}^{\mathbf{k}(1)}(t, \tau)+O\left(\epsilon^{2}\right)
$$

Up to the first order of $\epsilon$, the derivatives with respect to the time scale $t$ are given by

$$
\begin{aligned}
& \dot{c}_{\mathbf{m}}^{\mathbf{k}}=\partial_{t} c_{\mathbf{m}}^{\mathbf{k}(0)}+\epsilon\left(\partial_{\tau} c_{\mathbf{m}}^{\mathbf{k}(0)}+\partial_{t} c_{\mathbf{m}}^{\mathbf{k}(1)}\right) \\
& \ddot{c}_{\mathbf{m}}^{\mathbf{k}}=\partial_{t}^{2} c_{\mathbf{m}}^{\mathbf{k}(0)}+\epsilon\left(2 \partial_{\tau} \partial_{t} c_{\mathbf{m}}^{\mathbf{k}(0)}+\partial_{t}^{2} c_{\mathbf{m}}^{\mathbf{k}(1)}\right) .
\end{aligned}
$$

Let us substitute these into the equation (11). Then, we see that our original ordinary differential equation is replaced by a partial differential equation. It may appear that the problem has been complicated. But, as will be seen below, there are many advantages of this method. For the zeroth order of $\epsilon$, we obtain a well known equation:

$$
\ddot{c}_{\mathbf{m}}^{\mathbf{k}(0)}+\omega_{\mathbf{m}}^{2} c_{\mathbf{m}}^{\mathbf{k}(0)}=0 .
$$

The solution is given by

$$
c_{\mathbf{m}}^{\mathbf{k}(0)}=B_{\mathbf{m}}^{\mathbf{k}}(\tau) e^{i \omega_{\mathbf{m}} t}+C_{\mathbf{m}}^{\mathbf{k}}(\tau) e^{-i \omega_{\mathbf{m}} t},
$$

where $B_{\mathbf{m}}^{\mathbf{k}}(\tau)=B_{m_{x} m_{y} m_{z}}^{k_{x} k_{y} k_{z}}(\tau), C_{\mathbf{m}}^{\mathbf{k}}(\tau)=C_{m_{x} m_{y} m_{z}}^{k_{x} k_{y} k_{z}}(\tau)$ are small time varying functions. The initial conditions are given by

$$
C_{\mathbf{m}}^{\mathbf{k}}(\tau=0)=\frac{1}{\sqrt{2 \omega_{\mathbf{k}}}} \delta_{\mathbf{k}, \mathbf{m}} ; \quad B_{\mathbf{m}}^{\mathbf{k}}(\tau=0)=0
$$

To first order in $\epsilon$, we get

$$
\ddot{c}_{\mathbf{m}}^{\mathbf{k}(1)}+\omega_{\mathbf{m}}^{2} c_{\mathbf{m}}^{\mathbf{k}(1)}=\delta_{n_{z} m_{z}}\left(8 \sum_{n_{x}} \sum_{n_{y}} g_{n_{y} m_{y} m_{x}}^{n_{n}} \Theta_{\mathbf{n}}^{\mathbf{k}(0)}+\sum_{n_{x}} g_{n_{x} m_{x}} \Theta_{\mathbf{n}}^{\mathbf{k}(0)} \delta_{n_{y} m_{y}}-\sum_{n_{y}} g_{n_{y} m_{y}} \Theta_{\mathbf{n}}^{\mathbf{k}(0)} \delta_{n_{x} m_{x}}\right)-2 \partial_{t} \partial_{\tau} c_{\mathbf{m}}^{\mathbf{k}(0)}
$$

Let us substitute the definition (12) with (8) and the zeroth order solution (16) into the right hand side of the equation (18) and then use the following relations: $2 i \sin \Omega t=\left(e^{i \Omega t}-e^{-i \Omega t}\right), 2 \cos \Omega t=\left(e^{i \Omega t}+e^{-i \Omega t}\right)$. It can be seen that 
the right hand side contains terms that produce secular terms. For a uniform expansion, these secular terms must vanish. In other words, any term with $e^{ \pm i \omega_{\mathbf{m}} t}$ on the right-hand side must vanish. If not, these terms would be in resonance with the left-hand side term and secularities would appear. After imposing the requirement that no term $e^{\mp i \omega_{\mathbf{m}} t}$ appear, we get the equations for $B_{\mathbf{m}}^{\mathbf{k}}(\tau), \quad C_{\mathbf{m}}^{\mathbf{k}}(\tau)$. Although there are infinitely many terms in the summations, only a few modes are coupled to each other because of the nonequidistant character of the spectrum. There are only a few positive integers $n_{x}, n_{y}$ that produce secular terms in (18) for a given external frequency $\Omega$.

As an illustration, we will give a specific example. Assume that the inter-mode coupling occurs between (1,1,1) and $(1,2,1),(2,1,1)$. Note that the external frequency $\Omega$ should be determined in such a way that these modes are coupled to each other. So, choose $\Omega=(\sqrt{3}+\sqrt{6}) \pi / L$. As a result, a set of equations for $B_{\mathbf{m}}^{\mathbf{k}}$ and $C_{\mathbf{m}}^{\mathbf{k}}$ for these modes are given by

$$
\begin{aligned}
\partial_{\tau} B_{111}^{k_{x} k_{y} k_{z}}-G_{121}^{111} C_{121}^{k_{x} k_{y} k_{z}}+G_{211}^{111} C_{211}^{k_{x} k_{y} k_{z}} & =0 \\
\partial_{\tau} C_{111}^{k_{x} k_{y} k_{z}}-G_{121}^{111} B_{121}^{k_{x} k_{y} k_{z}}+G_{211}^{111} B_{211}^{k_{x} k_{y} k_{z}} & =0 \\
\partial_{\tau} B_{121}^{k_{x} k_{y} k_{z}}-G_{111}^{121} C_{111}^{k_{x} k_{y} k_{z}} & =0 \\
\partial_{\tau} C_{121}^{k_{x} k_{y} k_{z}}-G_{111}^{121} B_{111}^{k_{x} k_{y} k_{z}} & =0 \\
\partial_{\tau} B_{211}^{k_{x} k_{y} k_{z}}+G_{111}^{211} C_{111}^{k_{x} k_{y} k_{z}} & =0 \\
\partial_{\tau} C_{211}^{k_{x} k_{y} k_{z}}+G_{111}^{211} B_{111}^{k_{x} k_{y} k_{z}} & =0
\end{aligned}
$$

where $G_{\mathbf{m}}^{\mathbf{k}}=\frac{\left(2 \omega_{\mathbf{m}}-\Omega\right) \Omega}{4 \omega_{\mathbf{k}}} g_{m_{i} k_{i}} \quad\left(i=x\right.$ if $m_{x} \neq k_{x}$ or $i=y$ if $\left.m_{y} \neq k_{y}\right)$.

The solutions can be obtained by solving the above six equations with the initial conditions (17). The solutions for $B_{\mathbf{m}}^{\mathbf{k}}(\tau)$ is given by

$$
\left(\begin{array}{c}
B_{111}^{211} \\
B_{111}^{121} \\
B_{121}^{111} \\
B_{211}^{111}
\end{array}\right)=\frac{1}{\lambda}\left(\begin{array}{r}
-G_{211}^{111} / \sqrt{2 \omega_{211}} \\
G_{121}^{111} / \sqrt{2 \omega_{121}} \\
G_{111}^{121} / \sqrt{2 \omega_{111}} \\
-G_{111}^{211} / \sqrt{2 \omega_{111}}
\end{array}\right) \quad \sinh (\lambda \tau)
$$

where $\lambda^{2}=G_{121}^{111} G_{111}^{121}+G_{211}^{111} G_{111}^{211}$.

Assume that the cavity returns to its initial orientation at time $t_{f}$ and stops rotating. The number of generated scalar particles in the mode $\left(m_{x}, m_{y}, m_{z}\right)$ are calculated using the following formula [8, 31].

$$
<N_{\mathbf{m}}>=\sum_{\mathbf{k}} 2 \omega_{\mathbf{m}}\left|B_{\mathbf{m}}^{\mathbf{k}}\right|^{2}
$$

Hence, we find the average number of scalar particles generated in the modes $(1,1,1),(1,2,1)$ and $(2,1,1)$.

$$
<N_{111}>=2<N_{121}>=2<N_{211}>=\sinh ^{2}\left(\lambda \tau_{f}\right)
$$

They increase exponentially in time. The similar result has been obtained for the dynamical Casimir effect problem [30, 31]. There it was assumed that the mirrors of the cavity oscillate in time.

\section{DISCUSSION}

So far in the literature, the dynamical Casimir effect problem has been studied only for the cavity whose mirror(s) is(are) in oscillatory motion. Here, we have investigated the same effect for the cavity which rotates around itself. It was theoretically suggested that the particles are generated from the rectangular cavity whose mirror is moving. Here we have shown that the particles are generated from the rotating rectangular cavity as well.

If the cavity is rotated at a constant acceleration, no resonance effect takes place. Resonance particle generation occurs if the angle $\theta(t)$ changes sinusoidally (8). For the translational dynamical Casimir effect problem, that occurs when the mirror accelerates sinusoidally (1).

The boundary conditions become time-dependent as the cavity rotates. We have transformed the time-dependent boundary conditions to the time-independent ones. Hence, we are left with a modified equation in the rotating coordinate system (9). We have used the Multiple Scale Analysis to find the number of generated particles. As an example, we have calculated it for the specific coupled modes $(1,1,1)$ and $(1,2,1),(2,1,1)$. We have found that the average number of generated particles increases exponentially in time (22). The same result was obtained for the 
translational dynamical Casimir effect problem. In other words, the formula for the number of generated particles are the same for both the rotating cavity and the cavity whose one mirror moves according to the equation (1).

Here, we have considered the scalar particle generation. The vector particle generation can also be studied for the rotating cavity. This problem is more difficult since the vector components are mixed as the rectangular cavity rotates. The generated photons have not fixed polarization as they have in the translational dynamical Casimir effect problem. So, multiple scale analysis may not work for that problem since it enable us to find the number of generated photons in a given polarization.

* Electronic address: cyuce@anadolu.edu.tr

[1] H. B. G. Casimir, Proc. K. Ned. Akad. Wet. 51, 793 (1948).

[2] S. K. Lamoreaux, Phys. Rev. Lett. 78, 5 (1997).

[3] E. Sassaroli, Y. N. Srivastava and A. Widom, Phys. Rev. A 50, 1027 (1994).

[4] C. K. Law, Phys. Rev. Lett. 73, 1931 (1994).

[5] O. Meplan and C. Gignoux, Phys. Rev. Lett. 76, 408 (1996).

[6] C. K. Cole and W. C. Schieve, Phys. Rev. A 52, 4405 (1995).

[7] V. V. Dodonov, A. B. Klimov, and V. I. Man'ko, Phys. Lett. A 149, 225 (1990).

[8] V. V. Dodonov, Adv. Chem. Phys. 119, 309 (2001).

[9] P. Wegrzyn, J. Phys. B: At. Mol. Opt. Phys. 40, 2621 (2007).

[10] D. T. Alves and E. R. Granhen, Phys. Rev. A 77, 015808 (2008).

[11] Y. N. Srivastava, A. Widom, S. Sivasubramanian and M. P. Ganesh, Phys. Rev. A 74, 032101 (2006).

[12] Woo-Joong Kim, J. H. Brownell, R. Onofrio, Phys. Rev. Lett. 96, 200402 (2006)

[13] C. Braggio, et al., Europhys. Lett. 70, 754 (2005).

[14] S. D. Liberato, C. Ciuti, F. I. Carusotto Phys. Rev. Lett. 98, 103602 (2007).

[15] A. Lambrecht, M. -T. Jaekel, and S. Reynaud, Eur. Phys. J. D 395 (1998).

[16] Jeong-Young. Ji, Hyun-Hee. Jung and Kwang-Sup. Soh, Phys. Rev. A 57, 4952 (1998).

[17] D. A. R. Dalvit, F. D. Mazzitelli, Phys. Rev. A 59, 3049 (1999).

[18] M. Ruser Phys. Rev. A 73, 043811 (2006).

[19] V.V. Dodonov, J. Phys. A: Math Gen. 31, 9835 (1998).

[20] L. Ling, L. Bo-Zang, Chin. Phys. Lett. 19, 1061 (2002).

[21] D. F. Mundarain, P. A. M. Neto, Phys. Rev. A 57, 1379 (1998).

[22] A. Lambrecht, M. T. Jaekel, and S. Reynaud, Phys. Rev. Lett. 77, 615 (1996).

[23] C. Yuce, Z. Ozcakmakli, J. Phys. A: Math Theor. 41, 265401 (2008).

[24] M. Razavy and J. Terning, Phys. Rev. D 31, 307 (1985).

[25] G. Calucci, J. Phys. A 25, 3873 (1992).

[26] C. K. Law, Phys. Rev. A 49, 433 (1994).

[27] V. V.Dodonov and A. B. Klimov, Phys. Rev. A 53, 2664 (1996).

[28] A. B. Klimov and V. Altuzar, Phys. Lett. A 226, 41 (1997).

[29] Jeong-Young Ji, Hyun-Hee. Jung, Jong-Woong. Park, and Kwang-Sup. Soh, Phys. Rev. A 56, 4440 (1997).

[30] M. Crocce, D. A. R. Dalvit, F .D . Mazzitelli, Phys. Rev. A 66, 033811 (2002).

[31] M. Crocce, D. A. R. Dalvit and F. D. Mazzitelli, Phys. Rev. A 64, 013808 (2001).

[32] R. Schutzhold, G. Plunien, and G. Soff, Phys. Rev. A 65, 043820 (2002); G. Schaller, R. Schutzhold, G. Plunien, and G. Soff, Phys. Rev. A 66, 023812 (2002).

[33] M. Ruser, J. Opt. B 7, 100 (2005). 